\title{
LV-VE-4
}

\section{Robotic single port (plus one) unroofing of multiple liver cysts using the DaVinci SP system}

\author{
Joohyun LEE, Hye-Sung JO, Dong-Sik KIM, Young-Dong YU*
}

Department of Surgery, Korea University College of Medicine, Seoul, Korea

Introduction: Unroofing or marsupialization of liver cyst is performed for symptomatic case or growing cysts. Laparoscopic approach is widely used but few reports describe robotic approach for this procedure. Nowadays, single-port robotic surgery is adopted for various surgical procedures. We present a case of robotic single port (plus one) unroofing of multiple liver cysts using the DaVinci SP system.

Methods: A 53-year-old female was referred for multiple liver cysts which were diagnosed 6 years ago. Albeit asymptomatic, surgical resection was decided for enlarging cysts and subsequent pancreatic duct dilatation. Robotic port was inserted through transumbilical incision and an additional 5-mm-port was placed on the left side of the DaVinci SP system for energy device and suction/irrigation. Unroofing was done for most cysts and a drain was placed through the additional port.

Results: The operation time was 90 minutes, including 2 minutes of docking time and 57 minutes of console time, with negligible intraoperative blood loss. The drain was removed on the fifth postoperative day and the patient discharged on the ninth postoperative day without acute complication. Postoperative CT scan 3 months later confirmed decreased size of liver cysts and no evidence of pancreatic duct dilatation.

Conclusions: We report our experience of robotic single port (plus one) unroofing of multiple liver cysts using the DaVinci SP system. To our knowledge, this is the first report in Korea using the DaVinci SP system for unroofing of liver cyst. We believe that this approach is superior to laparoscopic approach while achieving similar safety outcome with fewer incisions. 